\title{
Mucoepidermoid carcinoma of the anterior lingual salivary gland: A rare case report
}

\author{
SHIMPEI GOTOH ${ }^{1}$, TOSHIYUKI NAKASONE ${ }^{1}$, AKIRA MATAYOSHI ${ }^{1}$, SHOKO MAKISHI $^{1}$, \\ FUSAHIRO HIRANO $^{1}$, EDWARD H. NTEGE ${ }^{1,2}$, YUSUKE SHIMIZU ${ }^{2}$ and HIROYUKI NAKAMURA ${ }^{1}$ \\ Departments of ${ }^{1}$ Oral and Maxillofacial Surgery; ${ }^{2}$ Plastic and Reconstructive Surgery, \\ Graduate School of Medicine, Ryukyu University, Nishihara, Okinawa 903-0215, Japan
}

Received May 14, 2021; Accepted August 26, 2021

DOI: $10.3892 / \mathrm{mco} .2021 .2444$

\begin{abstract}
Mucoepidermoid carcinomas (MECs) are rare head and neck malignant tumours that were originally considered to be benign. It has been estimated that $20 \%$ of MECs in the major salivary glands, such as the parotid gland, and $50 \%$ in the several minor salivary glands found in the oral cavity, are malignant. The diagnosis of MECs is mainly based on ancillary and immunohistochemistry testing. However, owing to the difficulty in harvesting adequate material for histological examination, the histopathological diagnosis of intraoral MECs may be particularly challenging. We herein report a rare case of an 82-year-old patient who presented to the Department of Oral and Maxillofacial Surgery of Ryukyu University Hospital with complaints of a progressive swelling and pain in the ventral surface of the apex of the tongue. The patient had previously undergone needle biopsy and the histopathological analysis of the tumour suggested a diagnosis of irritation fibroma. To ensure a more accurate histopathological assessment, an incisional biopsy was performed, in addition to the haematological and radiological assessments. Examination of the obtained surgical specimen confirmed low-grade MEC of the anterior lingual gland. The tumour was surgically excised, the patient healed uneventfully and no recurrence was detected on the regular 3-year follow-up. Although MECs are relatively more common in the minor salivary glands of the oral cavity, they are a rare occurrence in the anterior lingual
\end{abstract}

Correspondence to: Professor Hiroyuki Nakamura, Department of Oral and Maxillofacial Surgery, Graduate School of Medicine, Ryukyu University, 207 Uehara, Nishihara, Nakagami, Okinawa 903-0215, Japan

E-mail: hnak@me.com

Abbreviations: MEC, mucoepidermoid carcinoma; NB, needle biopsy; ${ }^{18}$ F-FDG-PET, fluorodeoxyglucose-positron emission tomography; STIR, short tau inversion recovery

Key words: head and neck tumour, mucoepidermoid carcinoma, minor salivary gland carcinoma, anterior lingual gland, low-grade tumour gland. Therefore, adequate histological material should be surgically harvested to perform a complete evaluation of the morphology and cytology of the tumour and ensure the accuracy of diagnosis.

\section{Introduction}

Mucoepidermoid carcinoma (MEC) is one of the most common primary salivary gland malignancies. MECs are characterised by mucin production, and they are composed of intermediate-type and squamoid cells exhibiting cystic and solid growth patterns (1). The tumour commonly develops in the three major salivary glands, including the parotid, submandibular and sublingual glands. Moreover, it can also develop in several minor salivary glands of the oral cavity, including glands of the tongue (lingual glands). The lingual glands are divided into various groups, among which the anterior lingual glands (glands of Blandin-Nuhn) are found near the ventral surface of the apex of the tongue. However, MECs of the anterior lingual glands are rarely reported (2-7).

Approximately 20 and $50 \%$ of the tumours that affect the major and minor glands, respectively, are malignant (1). The diagnosis of MECs is mainly based on H\&E staining of the tumours and a combination of both H\&E and immunohistochemistry tests, such as cytokeratin 5/6 (CK5/6) and transformation-related protein (p63) staining. To reduce the risk of misdiagnosis, sections stained with H\&E for histopathological diagnosis require adequate biopsy material in order to uncover the full range of morphological and cytological characteristics. We herein present a rare case of a patient with MEC of the anterior lingual gland, who initially underwent needle biopsy (NB) and was misdiagnosed using the obtained specimen. An alternative approach, namely the incisional biopsy, which was instrumental in confirming the diagnosis of low-grade MEC of the anterior lingual gland in the present case, is also discussed and the relevant literature is reviewed.

\section{Case report}

Patient history. An 82-year-old woman was referred to the Department of Oral and Maxillofacial Surgery of Ryukyu University Hospital in April 2018 from a private dental practitioner. The patient presented with a $\sim 5$-month history 
of swelling on the ventral surface of the apex of the tongue. The swelling was insidious in onset, had gradually increased to the current size and reportedly interfered with the patient's chewing. The initial (primary) assessment of the lesion was at a nearby hospital. Based on the patient's medical records, the primary assessment resulted in a working diagnosis of irritation fibroma following histopathological examination of a specimen obtained via NB. The histopathological review mentioned that the tongue mucosal tissue had thick collagen bundles interspersed with numerous capillaries and dotted chronic inflammatory cells under the surface of the squamous epithelium, with no findings suggestive of malignancy (Fig. S1). However, 2 weeks prior to the referral the patient suddenly developed pain at the tumour site, which worsened during eating, and decided to seek a second opinion. In addition, the patient had a history of hypertension and osteoporosis; however, the dental, social and family history was unremarkable. General physical examination indicated that the patient was moderately built and well-nourished, with no pallor, icterus, cyanosis or clubbing. The patient's face was symmetrical and there was no local or generalised lymphadenopathy. Intraoral examination revealed a round well-circumscribed mass, measuring 19x15 mm, in the right ventral surface of the apex of the tongue. Although the patient's tongue movement was impaired, there was no evidence of dysphagia (Fig. 1). Locally, the mass was tender on palpation and hard in consistency.

Investigations. Radiological assessment was performed, including contrast-enhanced CT scan, contrast-enhanced MRI using the short-tau inversion-recovery (STIR)-PROPELLER technique, and fluorodeoxyglucose-positron emission tomography $\left({ }^{18} \mathrm{~F}-\mathrm{FDG}-\mathrm{PET}\right) / \mathrm{CT}$, in addition to the routine haematological and histopathological diagnostic evaluations.

Axial CT scan revealed a metallic artefact that hindered a clear view of the right hypoglossal area. The MRI (Fig. 2A) and STIR-PROPELLER imaging (Fig. 2B) revealed right anterior lingual hyperintensities on diffusion-weighted imaging. Preoperative FDG-PET/CT examination showed abnormal FDG accumulation in the right anterior lingual margin (maximum standardized uptake value: 6.56; Fig. 3). None of the investigative findings were indicative of cervical lymph node or distant metastasis. The findings of the haematological evaluations were normal. The differential diagnosis included tongue cancer and mucous cyst.

Treatment and follow-up. The patient was locally anaesthetised without sedation, the tongue was stabilised with a traction suture and a perilesional (incisional) biopsy was performed to establish a definitive diagnosis, perform histological grading and select the appropriate treatment. On gross examination, the resected specimen included a poorly circumscribed lesion with a whitish appearance in the posterior region. Histopathological examination revealed that the superficial layer comprised tissue covered with thickened, ulcerated stratified squamous epithelium; atypical cells were arranged in solid sheets and alveoli and proliferated infiltratively. No obvious keratinisation was observed. On immunohistochemistry examination, the cells arranged in alveolar formations were positive for cytokeratin AE1/AE3 and carcinoembryonic antigen (data not shown), and the intra-alveolar mucus was positive for periodic acid-Schiff staining (Fig. 4A). The cells had irregular nuclei and foamy bright endoplasmic reticulum, and they proliferated in a vesicular nest and sheet-like pattern (Fig. 4B); furthermore, perineural invasion was also identified (Fig. 4C). Taken together, these findings confirmed the diagnosis of low-grade MEC following the histopathological grading system described by Goode et al (8). The TNM stage of the lesion was T1N0M0.

One month later, in April 2018, partial resection of the right anterior part of the patient's tongue was performed, with a $10-\mathrm{mm}$ safety margin around the tumour. Furthermore, split-thickness skin grafting was performed under general anaesthesia (Fig. 5). The patient's healing was uneventful (Fig. S2) and she was followed-up over a period of 3 years, with physical examinations performed monthly in the first year, bi-monthly in the second year and every 6 months in the third year (last follow-up visit, April 2021). An axial CT scan of the oral cavity performed 1 year postoperatively did not indicate any evidence of recurrence or metastasis (Fig. S3).

\section{Discussion}

First described in 1945 by Stewart et al (9), MEC commonly affects adults in their fifth and sixth decades of life (10), although it exhibits a predilection for individuals in their 30 s and 40s (11) and those of female sex (12). Although MEC accounts for $30 \%$ of all cancers of the salivary glands, it comprises $<5 \%$ of head and neck cancers. An estimated $50-60 \%$ of these tumours arise in the major salivary glands, with $>80 \%$ occurring in the parotid gland, $8-13 \%$ in the submandibular gland and $2-4 \%$ in the sublingual gland. The remaining $20 \%$ occurs in minor salivary glands, mostly in the palate, with the tongue being the least frequently affected site $(10,13)$. MECs can develop in any of the salivary glands in the three parts of the tongue: The anterior lingual glands (glands of Blandin-Nuhn) under the ventral surface of the apex of the tongue, the Ebner's gland in the submucosa of the foliate and circumvallate papillae, and the posterior lingual gland in the base of the tongue and the lingual margin. Moreover, among the three parts of the tongue, most malignancies reportedly occur in the base (14). In the present case, the lesion was detected under the right ventral surface of the apex of the tongue, near the lingual frenulum (a site of opening of the duct of the anterior lingual glands); thus, it was considered to have originated from the anterior lingual salivary gland.

It has been emphasised that the histopathological diagnosis of MEC is primarily based on morphology and ancillary staining in combination with immunohistochemistry (15). However, it may be challenging, particularly in high-grade tumours and intraoral MECs comprising prominent clear cells and a significant amount of oncocytic material (Warthin-like variant) in small biopsy specimens (15). In the present case, a NB was first performed elsewhere, and fibrous connective tissue, collagen bundles interspersed with numerous capillaries and chronic inflammatory cells under the surface of squamous epithelium were identified on histological examination. Indeed, these findings are suggestive of an irritation fibroma. The details of the NB procedure at the primary health care 


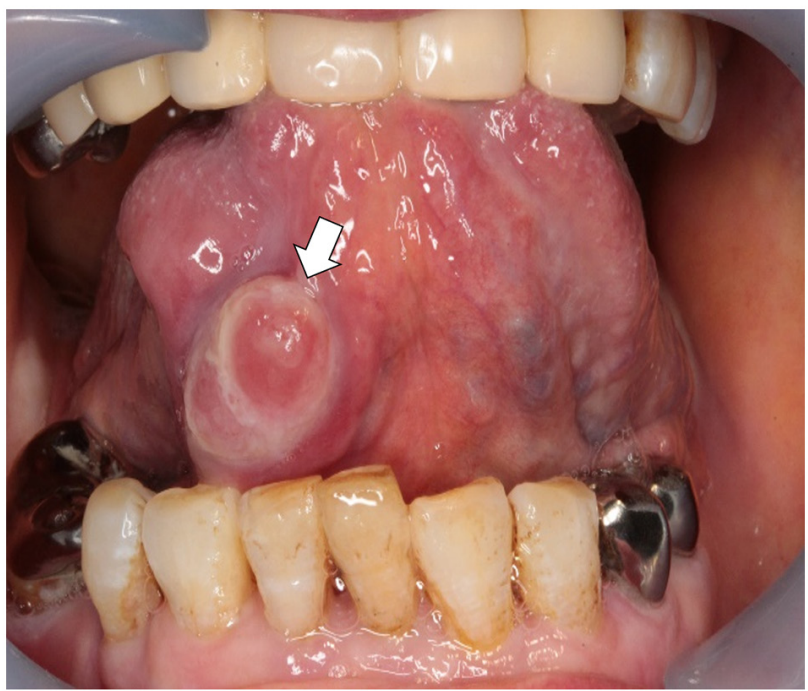

Figure 1. Macroscopic appearance of the lesion. A well-circumscribed mass (arrow) was located near the ventral surface of the apex of the tongue, measuring 19x15 mm.
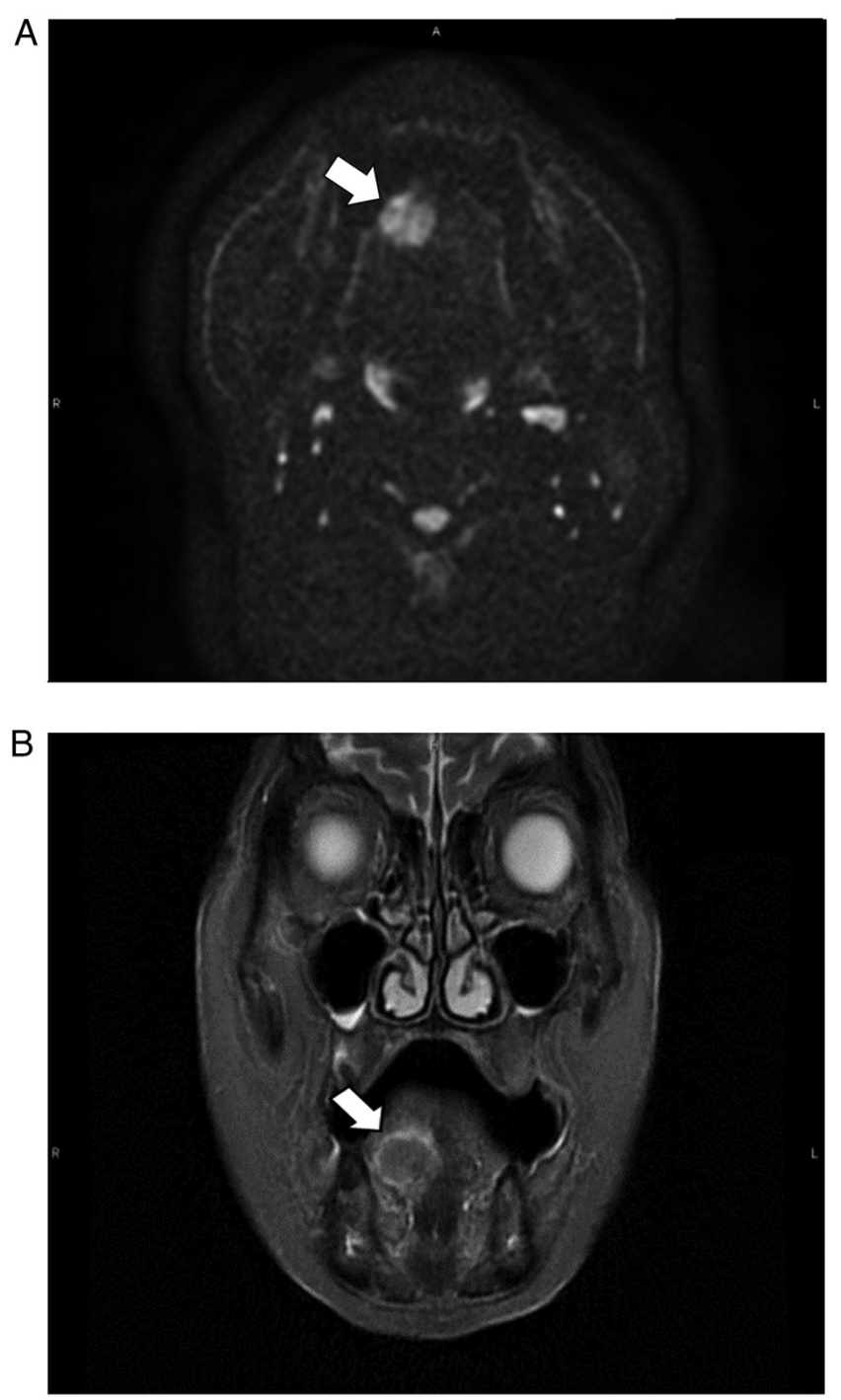

Figure 2. MRI examination of the lesion revealed (A) high signal intensity (arrow) on diffusion-weighted imaging and (B) a similarly hyperintense lesion (arrow) using the short tau inversion recovery-PROPELLER imaging technique.

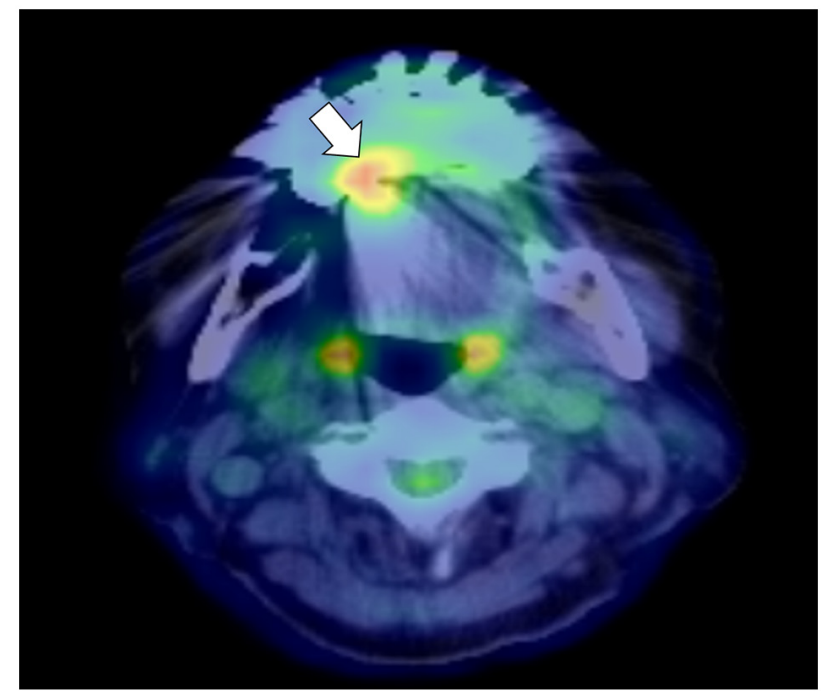

Figure 3. Imaging examination using ${ }^{18} \mathrm{~F}-\mathrm{FDG}$-positron emission tomography revealed high FDG accumulation (arrow) consistent with the lesion in the right ventral surface of the apex of the tongue. ${ }^{18} \mathrm{~F}$-FDG, fluorodeoxyglucose.

facility were not clear. However, it may be inferred that either the NB was taken from scarred and inflamed tissue around the tumour or the procedure was poorly performed and an inadequate amount of tissue was collected. Accurate preoperative diagnosis enables the formulation of a treatment plan that is appropriate for the disease grade. The initial symptom experienced in the present case was discomfort during eating due to the swelling. The patient later developed pain on chewing. This likely prompted the patient to seek a second opinion from the private dental practitioner, who then referred her to our hospital, leading to detection of the cancer at the relatively early stage of T1N0M0.

Goode et al (8) evaluated tumours histologically by the presence of specific parameters, such as $<20 \%$ intracystic component, neural invasion, necrotic foci, a mean of $\geq 4$ mitoses per 10 high-power fields and anaplasia, and then graded them accordingly using a score ranging from 0 to 14 as follows: 0-4, low-grade; 5-6, intermediate-grade; and $\geq 7$, high-grade. Treatment recommendations can be made based on this scoring; for example, soft tissue resection with a 1-cm mucosal margin should be performed for T1 low-grade MEC without clinical or radiographic signs of osseous invasion $(16,17)$. For the majority of the patients, wide surgical excision is usually sufficient; however, adjuvant therapy is indicated in cases with high-grade MEC, tumour-positive margins or evidence of lymph node infiltration, or residual disease (18). McHugh et al (19) stated that surgical resection is the treatment of choice for MEC, and surgical margins are considered important for preventing recurrence. In patients with close margins after resection, re-excision is preferred for intraoral tumours as opposed to adjuvant irradiation, as the latter is associated with significant morbidity (20). Brandwein et al (21) reported that none of the patients undergoing re-excision developed tumour recurrence, and were thus classified as having negative margins $(>3 \mathrm{~mm})$. Some authors recommend a prophylactic neck dissection for high-grade and clinical T3 or T4 tumours (22). Elective neck irradiation may be appropriate for patients with an 
A

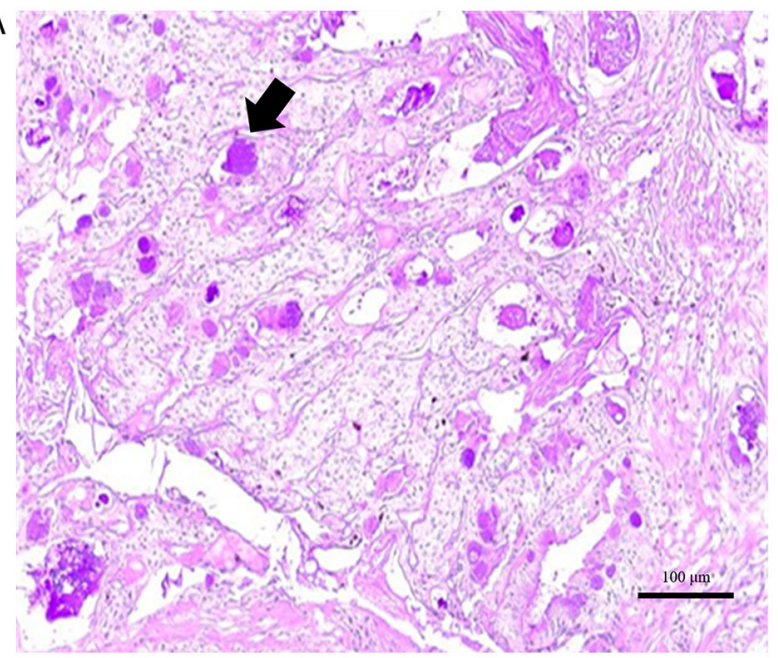

B
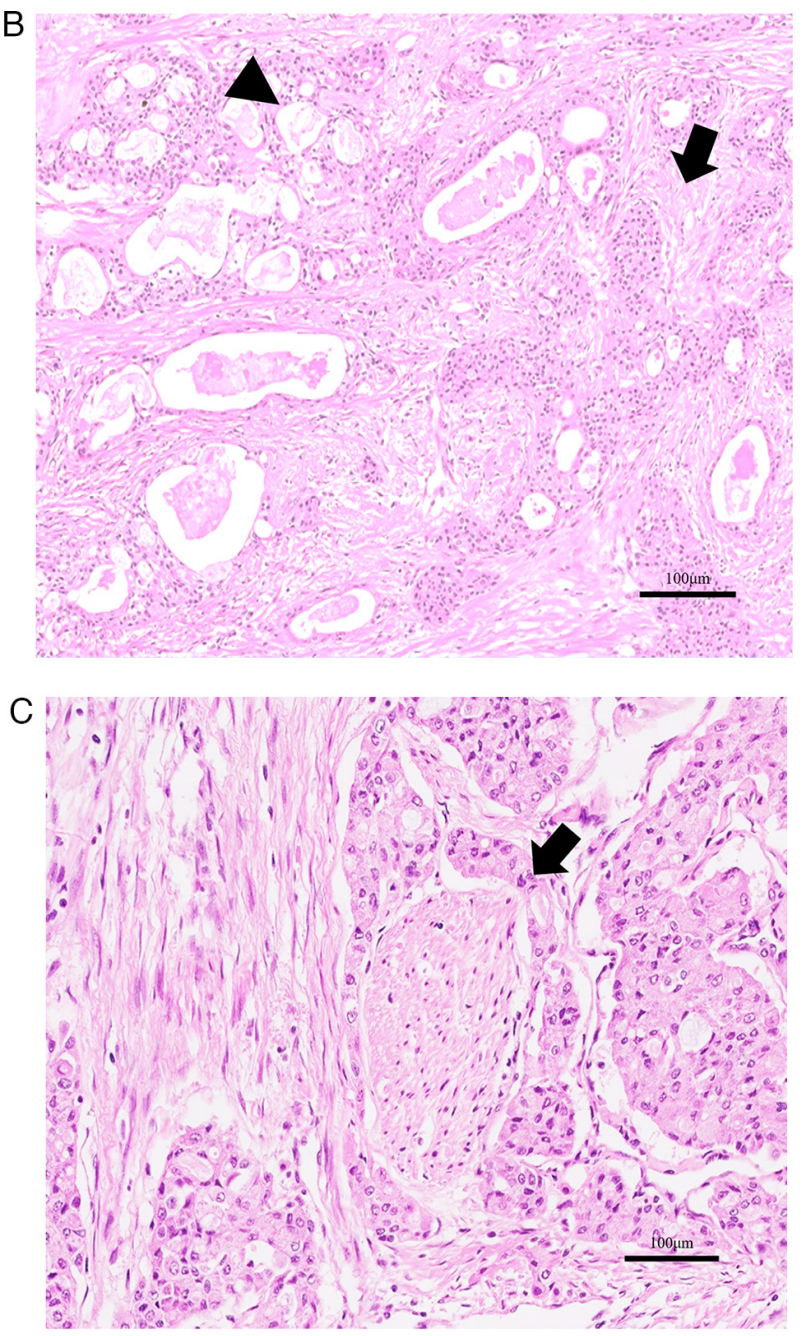

Figure 4. Histopathological examination of the incisional biopsy sections On PAS staining, (A) PAS-positive intra-alveolar mucus was identified (arrow); bar, $100 \mu \mathrm{m}$. (B) On H\&E staining, cells with irregularly shaped nuclei and foamy bright endoplasmic reticulum proliferated in a vesicular nest (arrowhead) and sheet-like pattern (arrow); bar, $100 \mu \mathrm{m}$. (C) Perineural invasion was also identified (arrow); bar, $100 \mu \mathrm{m}$. PAS, periodic acid-Schiff.

increased risk of nodal metastases (17). Spiro et al (23) reported that, among 367 cases of MEC, 59\% of high-grade, $30 \%$ of intermediate-grade and $7 \%$ of low-grade tumours had cervical lymph node metastasis. Evans (24) examined

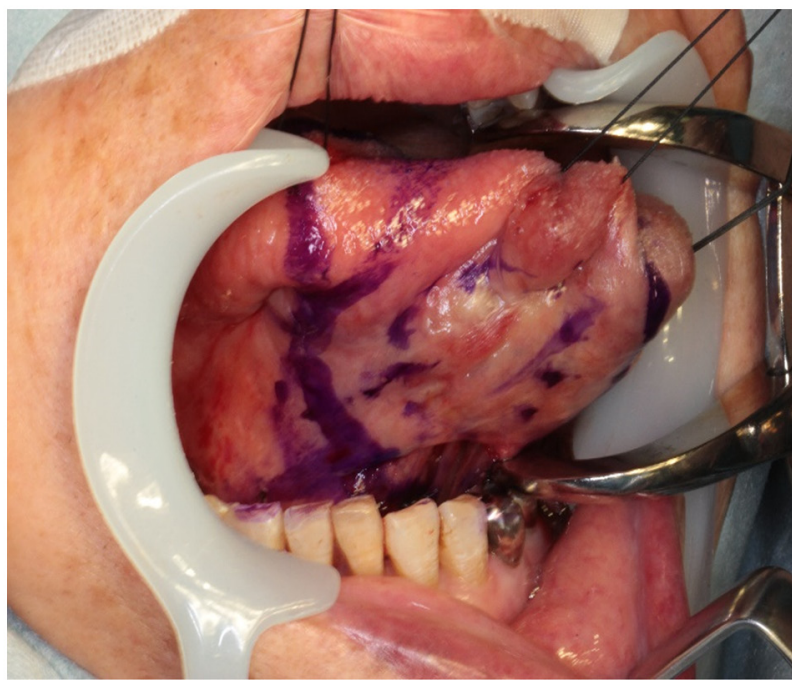

Figure 5. Intraoperative photograph during surgical excision of the tumour. A $10-\mathrm{mm}$ safety margin was drawn around the tumour to indicate the incision line.

69 cases and found that $70 \%(14 / 20)$ of high-grade and $8 \%$ (4/49) of low-grade tumours were associated with cervical lymph node metastasis. Hicks et al (25), in their study on 48 MEC cases, reported lymph node metastasis in $72 \%$ of high-grade, $22 \%$ of intermediate-grade and $0 \%$ of low-grade tumours. Based on these results, the frequency of cervical lymph node metastasis was closely associated with the tumour grade. MECs of the minor salivary glands have been reported to be most commonly low-grade and to have a better clinical course compared with MECs of the major salivary glands $(6,8,26)$.

In the present case, the patient was diagnosed with low-grade MEC as per the histological classification of Goode et al (8), in which the biopsy scored 4 points and a safety margin of $10 \mathrm{~mm}$ was set for the operation. Although no signs of recurrence were observed in this patient 3 years postoperatively, there are reports of local recurrence and distant metastasis that warrant the continuation of follow-up, even after this period (8).

In conclusion, we herein presented a rare case of an initially misdiagnosed low-grade MEC of the anterior lingual gland, which was correctly diagnosed using an alternative approach to the NB, the incisional biopsy. Tumours that develop in rarely affected sites, such as the anterior lingual glands, should be carefully investigated, and sufficient histological specimens should be obtained to ensure early detection of malignancy, accurate tumour grading and treatment optimization. The incisional biopsy is likely superior to NB for obtaining adequate material for an accurate diagnostic histopathological evaluation.

\section{Acknowledgements}

Not applicable.

\section{Funding}

No funding was received. 


\section{Availability of data and materials}

The datasets used and/or analysed for the case report are available from the corresponding author on reasonable request.

\section{Authors' contributions}

SG, TN, SM and FH analyzed and interpreted the patient data; SG, AM and EHN drafted and critically revised the manuscript for important intellectual content; YS and HN confirmed the authenticity of all the raw data and provided final approval of the completed article. All the authors read and approved the final manuscript.

\section{Ethics approval and consent to participate}

Not applicable.

\section{Patient consent for publication}

Written informed consent was obtained from the patient for both the surgical treatment and publication of the case details and any accompanying images.

\section{Competing interests}

The authors declare that they have no competing interests.

\section{References}

1. Lin HH, Limesand KH and Ann DK: Current state of knowledge on salivary gland cancers. Crit Rev Oncog 23: 139-151, 2018

2. Mathew L, Janardhanan M, Suresh R and Savithri V: Mucoepidermoid carcinoma of the posterior-lateral border of tongue: A rare presentation. BMJ Case Rep 2017: bcr2017221521, 2017.

3. Zahran M and Youssef A: Mucoepidermoid carcinoma of the tongue base: A case report. OTO Open 2: 2473974x18791559, 2018

4. Mesolella M, Iengo M, Testa D, DI Lullo AM, Salzano G and Salzano FA: Mucoepidermoid carcinoma of the base of tongue. Acta Otorhinolaryngol Ital 35: 58-61, 2015.

5. Goldblatt LI and Ellis GL: Salivary gland tumors of the tongue. Analysis of 55 new cases and review of the literature. Cancer 60: 74-81, 1987.

6. Auclair PL, Goode RK and Ellis GL: Mucoepidermoid carcinoma of intraoral salivary glands. Evaluation and application of grading criteria in 143 cases. Cancer 69: 2021-2030, 1992.

7. Foote FW Jr and Frazell EL: Tumors of the major salivary glands. Cancer 6: 1065-1133, 1953.

8. Goode RK, Auclair PL and Ellis GL: Mucoepidermoid carcinoma of the major salivary glands: Clinical and histopathologic analysis of 234 cases with evaluation of grading criteria. Cancer 82: 1217-1224, 1998.

9. Stewart FW, Foote FW and Becker WF: Muco-epidermoid tumors of salivary glands. Ann Surg 122: 820-844, 1945.
10. Pires FR, Chen SY, da Cruz Perez DE, de Almeida OP and Kowalski LP: Cytokeratin expression in central mucoepidermoid carcinoma and glandular odontogenic cyst. Oral Oncol 40: 545-551, 2004.

11. Chaudhry AP, Vickers RA and Gorlin RJ: Intraoral minor salivary gland tumors. An analysis of 1,414 cases. Oral Surg Oral Med Oral Pathol 14: 1194-1226, 1961.

12. Gill S, Mohan A, Aggarwal S and Varshney A: Mucoepidermoid carcinoma of hard palate. Indian J Pathol Microbiol 61: 397-398, 2018.

13. Devaraju R, Gantala R, Aitha H and Gotoor SG: Mucoepidermoid carcinoma. BMJ Case Rep 2014: bcr-2013-202776, 2014.

14. Burbank PM, Dockerty MB and Devine KD: A clinicopathologic study of 43 cases of glandular tumors of the tongue. Surg Gynecol Obstet 109: 573-582, 1959.

15. Moutasim KA and Gareth JT: Salivary gland tumours: Update on molecular diagnostics. Diagnostic Histopathol 26: 159-164, 2020.

16. Yih WY, Kratochvil FJ and Stewart JC: Intraoral minor salivary gland neoplasms: Review of 213 cases. J Oral Maxillofac Surg 63: 805-810, 2005

17. Chen AM, Garcia J, Lee NY, Bucci MK and Eisele DW: Patterns of nodal relapse after surgery and postoperative radiation therapy for carcinomas of the major and minor salivary glands: What is the role of elective neck irradiation? Int J Radiat Oncol Biol Phys 67: 988-994, 2007.

18. Liu S, Ow A, Ruan M, Yang W, Zhang C and Wang L: Prognostic factors in primary salivary gland mucoepidermoid carcinoma: An analysis of 376 cases in an Eastern Chinese population. Int J Oral Maxillofac Surg 43: 667-673, 2014.

19. McHugh CH, Roberts DB, El-Naggar AK, Hanna EY, Garden AS, Kies MS, Weber RS and Kupferman ME: Prognostic factors in mucoepidermoid carcinoma of the salivary glands. Cancer 118: 3928-3936, 2012.

20. Yan K, Yesensky J, Hasina R and Agrawal N: Genomics of mucoepidermoid and adenoid cystic carcinomas. Laryngoscope Investig Otolaryngol 3: 56-61, 2018.

21. Brandwein MS, Ivanov K, Wallace DI, Hille JJ, Wang B, Fahmy A, Bodian C, Urken ML, Gnepp DR, Huvos A, et al: Mucoepidermoid carcinoma: A clinicopathologic study of 80 patients with special reference to histological grading. Am J Surg Pathol 25: 835-845, 2001.

22. Ellis MA, Graboyes EM, Day TA and Neskey DM: Prognostic factors and occult nodal disease in mucoepidermoid carcinoma of the oral cavity and oropharynx: An analysis of the National Cancer Database. Oral Oncol 72: 174-178, 2017.

23. Spiro RH, Huvos AG, Berk R and Strong EW: Mucoepidermoid carcinoma of salivary gland origin. A clinicopathologic study of 367 cases. Am J Surg 136: 461-468, 1978.

24. Evans HL: Mucoepidermoid carcinoma of salivary glands: A study of 69 cases with special attention to histologic grading. Am J Clin Pathol 81: 696-701, 1984.

25. Hicks MJ, el-Naggar AK, Flaitz CM, Luna MA and Batsakis JG: Histocytologic grading of mucoepidermoid carcinoma of major salivary glands in prognosis and survival: A clinicopathologic and flow cytometric investigation. Head Neck 17: 89-95, 1995.

26. Melrose RJ, Abrams AM and Howell FV: Mucoepidermoid tumors of the intraoral minor salivary glands: A clinicopathologic study of 54 cases. J Oral Pathol 2: 314-325, 1973.

(1) (3) This work is licensed under a Creative Commons Attribution-NonCommercial-NoDerivatives 4.0 International (CC BY-NC-ND 4.0) License. 